\title{
DYNAMICAL ENTROPY \\ OF A NON-COMMUTATIVE VERSION OF THE PHASE DOUBLING
}

\author{
JOHAN ANDRIES and MIEKE DE COCK \\ Instituut voor Theoretische Fysica, K.U. Leuven \\ Celestijnenlaan 200D, 3001 Leuven, Belgium \\ E-mail: Johan.Andries@fys.kuleuven.ac.be, Mieke.DeCock@fys.kuleuven.ac.be
}

\begin{abstract}
A quantum dynamical system, mimicking the classical phase doubling map $z \mapsto$ $z^{2}$ on the unit circle, is formulated and its ergodic properties are studied. We prove that the quantum dynamical entropy equals the classical value $\log 2$ by using compact perturbations of the identity as operational partitions of unity.
\end{abstract}

1. Introduction. In the last few years a lot has been done in connection with the formulation of a quantum analogue of the classical Kolmogorov Sinai entropy (KS). This paper is meant as a step forward in the direction of a quantum dynamical entropy as proposed by R. Alicki and M. Fannes, based on an idea of Lindblad's [2]. This ALF entropy has been defined for automorphisms and up till now only invertible dynamics have been studied, e.g. the shift on a spin chain, the quantum Arnold cat map, ... One of our aims is to construct a non-commutative irreversible dynamics, mimicking the classical map $2 x$ modulo 1 on the unit interval, and to compute its entropy.

In the KS entropy construction the original dynamical system is mapped into a classical spin chain model by means of a partition of the phase space, referred to as coarse graining. The supremum, over all possible partitions, of the entropy density of these spin chains is then the KS invariant. For quantum systems a similar scheme can be performed by replacing the partitions of phase space by "operational partitions of unity". We will briefly sketch it in the preliminaries section.

As a consequence of the non-commutativity, the concept of partition must be treated with more care: not all partitions of unity will be allowed. To understand this, we have to look at the physical meaning of a partition of unity. In fact, asking for the proper partitions of unity is a mathematical matter which can be translated into physical terms

1991 Mathematics Subject Classification: Primary 46L55; Secondary 28D20.

M. De Cock is FWO-onderzoeker.

The paper is in final form and no version of it will be published elsewhere. 
by considering the question of which measurements are physically allowed. Indeed, a partition of unity is the theoretical expression of the coupling between the physical system and a measuring device (an array of quantum spins). For example, measurements that produce by themselves at a fixed rate a non-zero entropy should not be permitted. So a second motivation to start this work was to understand better which partitions can be allowed for in our simple model. It will turn out that the partitions that are only an "infinitesimal" perturbation of the trivial partition form a reasonable class, i.e. those partitions which differ only by compact operators from the trivial one.

Our starting point is the map

$$
\theta:[0,1[\rightarrow[0,1[: x \mapsto 2 x \bmod 1,
$$

leaving the Lebesgue measure invariant. This map is a standard example of a classical irreversible dynamical system and it is well known to be chaotic, which means that there exists a positive Lyapunov exponent $\lambda$, defined as

$$
\lambda:=\lim _{n \rightarrow \infty} \frac{1}{n} \log \left|\mathrm{D}_{x}\left(\theta^{n}(x)\right)\right|=\log 2 .
$$

By means of the shift

$$
T_{x_{0}}:\left[0,1\left[\rightarrow \left[0,1\left[: x \mapsto x+x_{0} \bmod 1\right.\right.\right.\right.
$$

we can formulate this notion in a frame suitable for an algebraic description:

$$
\theta \circ T_{x_{0}}=T_{2 x_{0}} \circ \theta .
$$

Formulated as such, it expresses the equivalence between first shifting a point over a distance $x_{0}$ and then applying the dynamics and, applying $\theta$ before shifting over twice the original distance.

It is our goal to put this map $\theta$ in a non-commutative, i.e. quantum framework. More precisely, we will be looking for an irreversible dynamics $\Theta$ on $\mathcal{B}\left(\mathcal{L}^{2}([0,1], d x)\right)$ satisfying

$$
\Theta\left(M_{f}\right)=M_{f \circ \theta},
$$

$M_{f}$ being the multiplication operator on $\mathcal{H}=\mathcal{L}^{2}([0,1], d x)$ by the function $f \in \mathcal{L}^{\infty}([0,1])$. Let us stress that we consider $\Theta$ as a dynamics on the whole of $\mathcal{B}(\mathcal{H})$, not only as a map on the multiplication operators. Requirement (2) by itself is not at all sufficient to determine a unique homomorphism. Therefore we will ask for extra properties.

Following the setup of [4] it is natural to impose for any $x_{0} \in[0,1[$

$$
\tau_{x_{0}} \circ \Theta=\Theta \circ \tau_{2 x_{0}}
$$

where $\tau_{x_{0}}$ is the automorphism implemented by the unitary operator

$$
\left(U_{x_{0}} \varphi\right)(x):=\varphi\left(T_{-x_{0}} x\right) \quad \varphi \in \mathcal{H} .
$$

Motivated by the observation that (3) restricted to the multiplication operators yields (1) again, we conclude that there exists a quantum Lyapunov exponent $\log 2$.

One could say that the dynamics stretches the position observable by a factor 2 . As $\Theta$ conserves the commutation relation between position and momentum it is to be expected that the momentum observable will shrink by a factor 2 under the dynamics. To see this, introduce the group of automorphisms $\left\{\sigma_{k} \mid k \in \mathbf{Z}\right\}$ determined by the unitaries 
$M_{\psi^{k}}$, where $\psi^{k}(x):=\exp (\mathrm{i} 2 \pi k x) . \sigma_{k}$ describes on the level of the observables a shift in momentum space. It follows then immediately from (2) that

$$
\sigma_{2 k} \circ \Theta=\Theta \circ \sigma_{k},
$$

showing the presence of a second Lyapunov exponent $-\log 2$.

In section 2 we will discuss the definition of dynamical entropy and we will say a few words about compact operators. Section 3 deals with the construction of the dynamics and its ergodic properties. In the last section the dynamical entropy is computed. Some results are stated without proof. For the details we refer to [3].

2. Preliminaries. We will begin this section by reminding the construction of the dynamical entropy for a discrete dynamical system [2] and stating a continuity property.

Let $\mathcal{M}$ be a von Neumann algebra of operators acting on a Hilbert Space $\mathcal{H}$ and let $\Omega$ be a normalized cyclic vector for $\mathcal{M}$, defining a state $\omega$ on $\mathcal{M}$. The single timestep evolution is given by an automorphism $\Theta$ (later on we will weaken this condition to a homomorphism) implemented by a unitary operator $U$ such that $U \Omega=\Omega$ and $U \mathcal{M} U^{*}=$ $\mathcal{M}$. In case a dynamical system is given in terms of a $C^{*}$-algebra one can make the GNS construction to obtain the von Neumann algebra picture.

An operational partition of unity of size $k$ is a $k$-tuple $\mathcal{X}$ of elements $x_{i} \in \mathcal{M}$ satisfying

$$
\sum_{i=0}^{k-1} x_{i}^{*} x_{i}=\mathbb{1}
$$

A partition $\mathcal{X}=\left(x_{0}, \ldots, x_{k-1}\right)$ evolves in time according to

$$
\Theta(\mathcal{X}):=\left(\Theta\left(x_{0}\right), \ldots, \Theta\left(x_{k-1}\right)\right) .
$$

It can be composed with another partition $\mathcal{Y}=\left(y_{0}, \ldots, y_{\ell-1}\right)$ to yield

$$
\mathcal{X} \circ \mathcal{Y}:=\left(x_{0} y_{0}, x_{0} y_{1}, \ldots, x_{k-1} y_{\ell-1}\right)
$$

which is of size $k \ell$.

To any partition $\mathcal{X}$ of size $k$ we associate a $k \times k$ density matrix $\rho[\mathcal{X}]$ with $(i, j)$ matrix element $\left\langle x_{j} \Omega, x_{i} \Omega\right\rangle$. The entropy $\mathrm{H}_{(\omega)}[\mathcal{X}]$ of the partition $\mathcal{X}$ is then

$$
\mathrm{H}_{(\omega)}[\mathcal{X}]:=\mathrm{S}(\rho[\mathcal{X}])=\mathrm{S}\left(\sum_{i=0}^{k-1}\left|x_{i} \Omega\right\rangle\left\langle x_{i} \Omega\right|\right),
$$

where the von Neumann entropy $\mathrm{S}(\rho)$ of a density matrix $\rho$ is computed as $\operatorname{Tr} \eta(\rho)$ with $\eta(0)=0$ and $\eta(x)=-x \log x$ for $0<x \leq 1$. Equality of the two von Neumann entropies is a consequence of the fact that both density matrices have, up to multiplicities of zero, identical spectrum. To see this, one has to consider the vector $\Psi_{\mathcal{X}}=\sum_{i} e_{i} \otimes$ $x_{i} \Omega,\left(e_{0}, \ldots, e_{k-1}\right)$ being a fixed orthonormal basis of $\mathbf{C}^{k}$. This vector is normalized and cyclic for $\mathcal{M}_{k} \otimes \mathcal{M}$. The restrictions of the pure vector state $\left|\Psi_{\mathcal{X}}\right\rangle\left\langle\Psi_{\mathcal{X}}\right|$ to $\mathcal{M}_{k}$ and $\mathcal{M}$ respectively are exactly $\rho[\mathcal{X}]$ and $\sum_{i}\left|x_{i} \Omega\right\rangle\left\langle x_{i} \Omega\right|$.

By composing the partition $\mathcal{X}$ with its subsequent time evolutions we can construct larger and larger density matrices $\rho\left[\Theta^{n-1}(\mathcal{X}) \circ \cdots \circ \Theta(\mathcal{X}) \circ \mathcal{X}\right]$ on $\mathcal{M}_{k}^{\otimes[0, n-1]}$. These are right-compatible for different $n$ in the sense that the partial trace over the last tensor factor, corresponding to time $n-1$, yields the density matrix up to time $n-2$. Therefore 
these matrices define a state $\omega_{\mathcal{X}}$ on $\mathcal{M}_{k}^{\otimes \mathbf{N}}$. The dynamical entropy $\mathrm{h}_{(\Theta, \omega)}[\mathcal{X}]$ of the partition $\mathcal{X}$ is then the mean entropy density of $\omega_{\mathcal{X}}$, i.e.

$$
\mathrm{h}_{(\Theta, \omega)}[\mathcal{X}]=\limsup _{n} \frac{1}{n} \mathrm{H}_{(\omega)}\left[\Theta^{n-1}(\mathcal{X}) \circ \cdots \circ \Theta(\mathcal{X}) \circ \mathcal{X}\right] .
$$

Consider a unital *-subalgebra $\mathcal{A}$ of $\mathcal{M}$ which is globally invariant under $\Theta$. The dynamical entropy $\mathrm{h}_{(\Theta, \omega, \mathcal{A})}$ is obtained by taking the supremum of the dynamical entropy over all finite partitions in $\mathcal{A}$ :

$$
\mathrm{h}_{(\Theta, \omega, \mathcal{A})}=\sup _{\mathcal{X} \subset \mathcal{A}} \mathrm{h}_{(\Theta, \omega)}[\mathcal{X}] .
$$

We finally state a continuity property of the entropy $\mathrm{H}_{(\omega)}[\mathcal{X}]$ of a partition.

LEmma 1. Consider two families $\mathcal{X}^{(\alpha)}=\left(x_{0}^{(\alpha)}, \ldots, x_{k-1}^{(\alpha)}\right)$ and $\mathcal{Y}^{(\alpha)}=\left(y_{0}^{(\alpha)}, \ldots, y_{k-1}^{(\alpha)}\right)$ of partitions, $\alpha=0, \ldots, n-1$, such that

Then

$$
\left\|x_{i}^{(\alpha)}-y_{i}^{(\alpha)}\right\|<\epsilon_{\alpha}(i=0, \ldots, k-1) \text { and } 2 k \sum_{\alpha=0}^{n-1} \epsilon_{\alpha}<\frac{1}{3} .
$$

for any state $\omega$.

$$
\begin{gathered}
\left|\frac{1}{n} \mathrm{H}_{(\omega)}\left[\mathcal{X}^{(n-1)} \circ \cdots \circ \mathcal{X}^{(0)}\right]-\frac{1}{n} \mathrm{H}_{(\omega)}\left[\mathcal{Y}^{(n-1)} \circ \cdots \circ \mathcal{Y}^{(0)}\right]\right| \\
\leq\left(2 k \sum_{\alpha=0}^{n-1} \epsilon_{\alpha}\right) \log (2 k)+\frac{1}{n} \eta\left(2 k \sum_{\alpha=0}^{n-1} \epsilon_{\alpha}\right)
\end{gathered}
$$

For the proof we refer to [6].

The norm closure of the ideal of finite rank operators on a Hilbert space $\mathcal{H}$ is the set of compact operators, $\mathcal{K}(\mathcal{H})$. These are the operators mapping uniformly bounded subsets of $\mathcal{H}$ into pre-compact sets. Each element $A$ of $\mathcal{K}(\mathcal{H})$ can be expressed in an essentially unique, norm convergent expansion

$$
A=\sum_{n \geq 1} \mu_{n}\left|\xi_{n}\right\rangle\left\langle\phi_{n}\right| .
$$

In this expression, the $\left\{\xi_{n}\right\}$ and $\left\{\phi_{n}\right\}$ are orthonormal sets and the $\mu_{n}>0$ are coefficients arranged in decreasing order. They are the non-zero eigenvalues of $|A|=U^{*} A$, with the $\phi_{n}$ the corresponding eigenvectors and $\xi_{n}=U \phi_{n}$. The lack of uniqueness comes from the possibility of degenerate eigenvalues of $|A|$.

For any $p \geq 1$ the Schatten class $\mathcal{L}_{p}$ is defined as

$$
\mathcal{L}_{p}=\left\{A \in \mathcal{K}(\mathcal{H}) \mid\|A\|_{p}=\left(\sum_{n \geq 1} \mu_{n}^{p}\right)^{1 / p}<\infty\right\} .
$$

For $p=1$ we get the trace class operators and $p=2$ corresponds to the Hilbert-Schmidt operators. Since $\left(\mu_{n}\right)_{n}$ is a decreasing sequence we can write

$$
N \mu_{N}^{p} \leq \sum_{n=1}^{N} \mu_{n}^{p}<\|A\|_{p}^{p}
$$

such that $\mu_{N} \leq\|A\|_{p} / N^{1 / p}$. This allows us to formulate: 
Lemma 2. For any $A \in \mathcal{L}_{p}$ and any $\epsilon>0$ there exists an operator $A_{N}$ of finite rank $N-1$ such that $\left\|A-A_{N}\right\| \leq \epsilon$ and

$$
N=\left[\left(\frac{\|A\|_{p}}{\epsilon}\right)^{p}\right],
$$

where $[x]$ denotes the smallest integer larger than $x$.

3. Construction of the dynamics. We propose the following form for the $*$-homomorphism $\Theta$ on $\mathcal{B}(\mathcal{H})$ that we are looking for:

$$
\Theta(A)=u_{0} A u_{0}^{*}+u_{1} A u_{1}^{*},
$$

where $u_{0}, u_{1} \in \mathcal{B}(\mathcal{H})$ satisfy the Cuntz relations

$$
u_{0} u_{0}^{*}+u_{1} u_{1}^{*}=\mathbb{I} \quad \text { and } \quad u_{0}^{*} u_{0}=u_{1}^{*} u_{1}=\mathbb{I} .
$$

Moreover, we want the homomorphism to act on the multiplication operators in the same way as the classical dynamics and we ask for a quantum Lyapunov exponent $\log 2$ (expressed by (2) and (3)).

Writing the first condition more explicitly, we have

$$
u_{0} M_{f} u_{0}^{*}+u_{1} M_{f} u_{1}^{*}=M_{f \circ \theta} .
$$

Multiplying this relation by $u_{0}$ on the right and using the Cuntz relations we find

$$
u_{0} M_{f}=M_{f \circ \theta} u_{0} .
$$

Let us denote the constant function with value 1 by $\mathbf{1}$. Applying the previous line to $\mathbf{1}$ and evaluating this at a point $x \in[0,1]$ we see that

$$
\left(u_{0} f\right)(x)=f(\theta(x))\left[u_{0}(\mathbf{1})\right](x) .
$$

Let $w \in \mathcal{B}(\mathcal{H})$ be the operator $(w \varphi)(x):=\varphi(\theta(x))$. A small computation shows that $w$ is an isometry: $w^{*} w=\mathbb{I}$. We use this to write $f=w^{*} g$ with $w f=g$. This implies

$$
\begin{aligned}
\left(u_{0} w^{*} g\right)(x) & =\left[u_{0}(\mathbf{1})\right](x)(w f)(x) \\
& =\left[u_{0}(\mathbf{1})\right](x) g(x) .
\end{aligned}
$$

From this, we can conclude that $u_{0}(\mathbf{1}) \in \mathcal{L}^{\infty}([0,1])$ and that $u_{0} w^{*}=M_{u_{0}(\mathbf{1})}$. A similar argument can be given for $u_{1}$ leading to the existence of two essentially bounded functions $f_{0}$ and $f_{1}$ such that

$$
u_{j}=M_{f_{j}} w \quad(j=0,1) .
$$

The fact that $\Theta$ is a unity preserving homomorphism will impose some conditions on $f_{0}$ and $f_{1}$.

Because the multiplication operators $M_{f}$ and the shifts $U_{x_{0}}$ generate a strongly dense subalgebra of $\mathcal{B}(\mathcal{H})$ and we consider strongly continuous homomorphisms, it is sufficient to check (3) for the $M_{f}$ and $U_{x_{0}}$. Using $\theta \circ T_{x_{0}}=T_{2 x_{0}} \circ \theta$, the relation

$$
\left(\tau_{x_{0}} \circ \Theta\right)\left(M_{f}\right)=\left(\Theta \circ \tau_{2 x_{0}}\right)\left(M_{f}\right)
$$

follows immediately. On the other hand

$$
\left(\tau_{x_{0}} \circ \Theta\right)\left(U_{x_{1}}\right)=\left(\Theta \circ \tau_{2 x_{0}}\right)\left(U_{x_{1}}\right)
$$


is, using the definition of $\tau_{x_{0}}$, equivalent to

$$
U_{x_{0}} \Theta\left(U_{x_{1}}\right)=\Theta\left(U_{x_{1}}\right) U_{x_{0}}
$$

for all $x_{0}, x_{1}$. Writing the shift $U_{x_{0}}$ in its spectral decomposition reads

$$
U_{x_{0}}=\sum_{k \in \mathbf{Z}} \mathrm{e}^{\mathrm{i} 2 \pi k x_{0}}\left|\psi^{k}\right\rangle\left\langle\psi^{k}\right| .
$$

The time evolved shift is then

$$
\begin{aligned}
\Theta\left(U_{x_{1}}\right) & =\sum_{k} e^{\mathrm{i} 2 \pi k x_{1}} \Theta\left(\left|\psi^{k}\right\rangle\left\langle\psi^{k}\right|\right) \\
& =\sum_{k} e^{\mathrm{i} 2 \pi k x_{1}}\left(\left|u_{0} \psi^{k}\right\rangle\left\langle u_{0} \psi^{k}|+| u_{1} \psi^{k}\right\rangle\left\langle u_{1} \psi^{k}\right|\right) \\
& =\sum_{k} e^{\mathrm{i} 2 \pi k x_{1}}\left(\left|M_{f_{0}} \psi^{2 k}\right\rangle\left\langle M_{f_{0}} \psi^{2 k}|+| M_{f_{1}} \psi^{2 k}\right\rangle\left\langle M_{f_{1}} \psi^{2 k}\right|\right)
\end{aligned}
$$

which is the spectral decomposition of $\Theta\left(U_{x_{1}}\right)$. If we want $U_{x_{0}}$ and $\Theta\left(U_{x_{1}}\right)$ to commute, they should have the same spectral projections which means that we have to impose $f_{0}=\psi^{l_{0}}$ and $f_{1}=\psi^{l_{1}}$. The earlier mentioned conditions on $f_{0}$ and $f_{1}$ are only valid iff $l_{0}$ an $l_{1}$ have different parity.

Writing the action of $u_{j}^{*}$ explicitly

$$
\left(u_{j}^{*} \varphi\right)(x)=\frac{1}{2} \exp \left(-\mathrm{i} \pi l_{j} x\right)\left(\varphi\left(\frac{x}{2}\right)+(-1)^{l_{j}} \varphi\left(\frac{x+1}{2}\right)\right) \quad(j=0,1),
$$

the validity of both imposed conditions is easily checked if $\Theta$ is of the derived form.

We have put the phase doubling in a non-commutative algebraic framework and we are also interested in the ergodic properties of the system. In order to arrive at a dynamical system with optimal ergodic and mixing properties, we will make a particular choice for the integers $l_{0}$ and $l_{1}$ in the definition of $u_{0}$ and $u_{1}$.

Consider therefore the inner product

$$
(\rho, A) \mapsto\langle\rho, A\rangle:=\operatorname{Tr} \rho A
$$

between the trace class operators $\mathcal{L}_{1}(\mathcal{H})$ and $\mathcal{B}(\mathcal{H})$. The map $\Theta$ has a pre-adjoint $\Theta_{*}$ which, from $\left\langle\Theta_{*}(\rho), A\right\rangle=\langle\rho, \Theta(A)\rangle$, is explicitly given by

$$
\Theta_{*}(\rho)=u_{0}^{*} \rho u_{0}+u_{1}^{*} \rho u_{1} \quad \rho \in \mathcal{L}_{1}(\mathcal{H}) .
$$

We are interested in density matrices invariant under $\Theta_{*}$ and, more generally, in the long time behaviour of the perturbed normal states $\omega_{\rho} \circ \Theta^{n}$. If we want the system to converge to an invariant state, we should avoid periodic behaviour. To obtain this, we have to exclude the case $\left|l_{0}-l_{1}\right|>1$. Indeed, we easily see that $\left|\psi^{-l_{0}}\right\rangle\left\langle\psi^{-l_{0}}\right|$ and $\left|\psi^{-l_{0}-1}\right\rangle\left\langle\psi^{-l_{0}-1}\right|$ are both invariant under $\Theta_{*}$ but suppose $\exists m: l_{0}<m<l_{1}$ with $l_{0}$ even. It is then easy to check that $\Theta_{*}\left(\left|\psi^{m}\right\rangle\left\langle\psi^{m}\right|\right)=\left|\psi^{r}\right\rangle\left\langle\psi^{r}\right|$ where $l_{0}<r<l_{1}$ and $r \neq m$. This implies that subsequent applications of $\Theta_{*}$ transform $\left|\psi^{m}\right\rangle\left\langle\psi^{m}\right|$ into itself via at least one intermediate $\left|\psi^{r}\right\rangle\left\langle\psi^{r}\right|\left(l_{0}<r<l_{1}\right)$.

In order to obtain a unique invariant state we consider the following projections

$$
P_{-}=\sum_{k \leq-l_{0}-1}\left|\psi^{k}\right\rangle\left\langle\psi^{k}\right| \text { and } P_{+}=\sum_{k \geq-l_{0}}\left|\psi^{k}\right\rangle\left\langle\psi^{k}\right|,
$$


such that $P_{-}+P_{+}=\mathbb{I}$. It takes a straightforward calculation to see that $u_{\epsilon}^{*} \psi^{k} \in P_{ \pm} \mathcal{H}$ as soon as $\psi^{k} \in P_{ \pm} \mathcal{H}(\epsilon=0,1)$, meaning that $P_{ \pm} \Theta(A) P_{ \pm}=\Theta(A)$ as soon as $P_{ \pm} A P_{ \pm}=A$. This allows us to consider only the subalgebra $\mathcal{A}$ of those operators for which $P_{+} A P_{+}=A$, or equivalently $\mathcal{B}\left(P_{+} \mathcal{H}\right)$. This restriction gives us the existence of the desired unique invariant state $\left|\psi^{-l_{0}}\right\rangle\left\langle\psi^{-l_{0}}\right|$. From now on we choose $l_{0}=0$ and hence $l_{1}=1$. This particular choice is quite convenient as it agrees well with the binary expansion of a natural number. All subsequent results will be independent of the value of $l_{0}$. Taking into account the foregoing remarks, we finally defined the following rather simple dynamical system: consider in $\ell^{2}(\mathbf{N})$ the canonical orthonormal basis $\left\{\psi^{0}, \psi^{1}, \ldots\right\}$ of sequences $\psi^{j}=$ $\left(\delta_{j n}\right)_{n \in \mathbf{N}}$ and the isometries $u_{0}$ and $u_{1}$ defined by

$$
\psi^{2 k+1}=0 \quad \text { and } \quad \psi^{2 k+1}=\psi^{k}
$$

for $k \in \mathbf{N}$. The single step time evolution on $\mathcal{A}=\mathcal{B}\left(\ell^{2}(\mathbf{N})\right)$ is given by

$$
A \mapsto u_{0} A u_{0}^{*}+u_{1} A u_{1}^{*} .
$$

The ergodic and mixing properties are stated in the following theorems and lemma (only the lemma is proved, the other proofs use the same techniques):

TheOREM 1. For any normal state $\omega_{\rho}$ on $\mathcal{A}$ given by $\omega_{\rho}(\cdot)=\operatorname{Tr}(\rho \cdot)$ we have

$$
\lim _{n \rightarrow \infty} \|\left(\Theta_{*}\right)^{n}(\rho)-\left|\psi^{0}\right\rangle\left\langle\psi^{0}\right| \|_{1}=0 \text {. }
$$

THEOREM 2. The spectrum of $\Theta$ with respect to the algebra $\mathcal{A}$ consists of the closed unit disc.

THEOREM 3. The only eigenvalues of $\Theta$ are 0 and 1 . Up to scalar multiples, the only eigenvector of $\Theta$ corresponding to the eigenvalue 1 is the unit operator.

Lemma 3. The pure state $\omega_{0}: A \mapsto\left\langle\psi^{0}, A \psi^{0}\right\rangle$ is mixing under $\Theta$, i.e.

$$
\lim _{n \rightarrow \infty} \omega_{0}\left(\Theta^{n}(A) B\right)=\omega_{0}(A) \omega_{0}(B)
$$

for any two operators $A, B \in \mathcal{A}$.

Pr o of. For any two operators $A$ and $B$ in $\mathcal{A}$, we have

$$
\begin{aligned}
\omega_{0}\left(\Theta^{n}(A) B\right) & =\sum_{\epsilon_{i_{n-1}}, \ldots, \epsilon_{i_{0}}=0,1}\left\langle\psi^{0}, u_{\epsilon_{i_{n-1}}} \ldots u_{\epsilon_{i_{0}}} A u_{\epsilon_{i_{0}}}^{*} \ldots u_{\epsilon_{i_{n-1}}}^{*} B \psi^{0}\right\rangle \\
& =\sum_{\epsilon_{i_{n-1}}, \ldots, \epsilon_{i_{0}}=0,1}\left\langle u_{\epsilon_{i_{0}}}^{*} \ldots u_{\epsilon_{i_{n}-1}}^{*} \psi^{0}, A u_{\epsilon_{i_{0}}}^{*} \ldots u_{\epsilon_{i_{n-1}}}^{*} B \psi^{0}\right\rangle .
\end{aligned}
$$

The left-hand side of the scalar product is different from zero iff $\epsilon_{i_{0}}=\ldots=\epsilon_{i_{n-1}}=0$ since $u_{0}^{*} \psi^{0}=\psi^{0}$ and $u_{1}^{*} \psi^{0}=0$ so

$$
\omega_{0}\left(\Theta^{n}(A) B\right)=\left\langle\psi^{0}, A u_{0}^{* n} B \psi^{0}\right\rangle=\sum_{k \geq 0}\left\langle\psi^{0}, A u_{0}^{* n} \psi^{k}\right\rangle\left\langle\psi^{k}, B \psi^{0}\right\rangle .
$$

$u_{0}^{* n} \psi^{k}$ will be different from zero only if $k$ is a multiple of $2^{n}$, say $k=k^{\prime} 2^{n}$. Using the 
fact that $u_{0}^{* n} \psi^{k^{\prime} 2^{n}}=\psi^{k^{\prime}}$ we get

$$
\omega_{0}\left(\Theta^{n}(A) B\right)=\sum_{k^{\prime} \geq 0}\left\langle\psi^{0}, A \psi^{k^{\prime}}\right\rangle\left\langle\psi^{k^{\prime} 2^{n}}, B \psi^{0}\right\rangle .
$$

So, separating out the term $k=0$, we see

$$
\begin{aligned}
\left|\omega_{0}\left(\Theta^{n}(A) B\right)-\omega_{0}(A) \omega_{0}(B)\right| & \leq \sum_{k \geq 1}\left|\left\langle\psi^{0}, A \psi^{k}\right\rangle\right|\left|\left\langle\psi^{2^{n} k}, B \psi^{0}\right\rangle\right| \\
& \leq\left(\sum_{k \geq 1}\left|\left\langle\psi^{0}, A \psi^{k}\right\rangle\right|^{2}\right)^{\frac{1}{2}}\left(\sum_{k \geq 1}\left|\left\langle\psi^{2^{n} k}, B \psi^{0}\right\rangle\right|^{2}\right)^{\frac{1}{2}} \\
& \leq\left\|A^{*} \psi^{0}\right\|\left(\sum_{k \geq 2^{n}}\left|\left\langle\psi^{k}, B \psi^{0}\right\rangle\right|^{2}\right)^{\frac{1}{2}}
\end{aligned}
$$

which tends to zero as $n$ goes to infinity.

4. Dynamical entropy. In this paragraph we compute the dynamical entropy of the introduced dynamics $\Theta$. As we mentioned before, we use this model to understand a bit more about which partitions are allowed. In particular, we will show that partitions generated by elements that are, up to compact perturbations, multiples of the identity, form a reasonable class. We prove that the dynamical entropy of the proposed homomorphism $\Theta$ equals the classical value if we consider partitions of this specific form.

In order to prove this result we show that $\log 2$ is an upperbound and that we can find a partition in which this bound is reached.

Before coming to the main result of this paper, we need a more technical lemma stating that every partition of unity of the above form can be approximated by another partition of unity of which the elements are finite rank perturbations of the identity-operator. The proof of this result is quite lengthy and technical. Therefore we only give the main idea.

Lemma 4. An operator $x=\alpha \mathbb{I}+K\left(\alpha \in \mathbf{C}, K \in \mathcal{L}_{p}\right)$ on a Hilbert space $\mathcal{H}$ can be written in the form $x=U|x|$ where $U$ is unitary and $U,|x| \in \mathbf{C} \mathbb{I}+\mathcal{L}_{p}$.

Lemma 5. For any partition of unity $\mathcal{X}=\left(x_{0}, \ldots, x_{k-1}\right) \subset \mathcal{B}(\mathcal{H})$ with elements of the form

$$
x_{i}=\alpha_{i} \mathbb{I}+K_{i} \quad \alpha_{i} \in \mathbf{C}, K_{i} \in \mathcal{L}_{p},
$$

there is a constant $C$ such that we can construct for every $\epsilon>0$ a partition $\mathcal{Y}$ with $\left\|x_{i}-y_{i}\right\|<\epsilon(i=0, \ldots, k-1)$. Furthermore $\mathcal{Y}$ is of the form

$$
y_{i}=\beta_{i} \mathbb{I}+\tilde{K}_{i} \quad \beta_{i} \in \mathbf{C}, \tilde{K}_{i} \text { finite rank }
$$

with $\tilde{K}_{i}=P_{\text {fin }} \tilde{K}_{i} P_{\text {fin }}$ where $P_{\text {fin }}$ is a projection of dimension

$$
N=2 k\left[\left(\frac{C}{\epsilon^{2}}\right)^{p}\right]
$$

at most.

Proof (sketch). The operators $\left(y_{0}, \ldots, y_{k-2}\right)$ are constructed by approximating the compact operators $K_{i}$, appearing in the $x_{i}$, by finite rank operators $\tilde{K}_{i}$. The rank of these operators can be controlled by lemma 2 . 
To construct $y_{k-1}$, we use lemma 4 to write

$$
x_{k-1}=U\left|x_{k-1}\right|=U \sqrt{\mathbb{I}-\sum_{i=0}^{k-2} x_{i}^{*} x_{i}},
$$

where $U=\mathrm{e}^{\mathrm{i} \theta_{0}} \mathbb{I}+L\left(L \in \mathcal{L}_{p}\right)$ is a unitary operator. Because of the unitarity of $U$, the structure of $L$ will be

$$
L=\sum_{n \geq 1}\left(\mathrm{e}^{\mathrm{i} \theta_{n}}-\mathrm{e}^{\mathrm{i} \theta_{0}}\right)\left|\varphi_{n}\right\rangle\left\langle\varphi_{n}\left|=\sum_{n \geq 1}\right| \mathrm{e}^{\mathrm{i} \theta_{n}}-\mathrm{e}^{\mathrm{i} \theta_{0}}|| \xi_{n}\right\rangle\left\langle\varphi_{n}\right|
$$

which gives us the canonical decomposition of $L$ provided that we arranged the $\varphi_{n}$ in such a way that $\left|\exp \left(\mathrm{i} \theta_{0}\right)-\exp \left(\mathrm{i} \theta_{n}\right)\right|$ is a decreasing sequence, converging to 0 . Approximating $L$ by a finite rank operator $\tilde{L}$, i.e. restricting the norm convergent sum to a finite number of terms, we get a new unitary $\tilde{U}=\exp \left(\mathrm{i} \theta_{0}\right) \mathbb{I}+\tilde{L}$. We now put

$$
y_{k-1}:=\tilde{U} \sqrt{\mathbb{I}-\sum_{i=0}^{k-2} y_{i}^{*} y_{i}} .
$$

Note that $\mathcal{Y}$ is a partition of unity because $\tilde{U}^{*} \tilde{U}=\mathbb{I}$. The full proof takes care of the fact that the argument of this square root could be non positive.

Lemma 6. For any partition of unity $\mathcal{X}=\left(x_{0}, \ldots, x_{k-1}\right)$ in $\mathcal{A}$ of the form

$$
x_{i}=\alpha_{i} \mathbb{I}+K_{i} \quad\left(\alpha_{i} \in \mathbf{C}, K_{i} \in \mathcal{L}_{p}\right),
$$

we have:

$$
\mathrm{h}_{\left(\Theta, \omega_{0}\right)}[\mathcal{X}] \leq \log 2 .
$$

Proof. Choose $\epsilon>0$ and consider the decreasing sequence $\epsilon_{\alpha}:=\epsilon /(\alpha+1)^{2}$. By taking $\epsilon$ sufficiently small we can make

$$
2 k \sum_{\alpha \geq 0} \epsilon_{\alpha}
$$

arbitrary small. Using lemma (5) we can find a sequence of partitions $\left(\hat{y}_{0}^{(\alpha)}, \ldots, \hat{y}_{k-1}^{(\alpha)}\right)$ (denoted by $\hat{\mathcal{Y}}^{(\alpha)}$ ) and a sequence of projections $\hat{P}_{\alpha}(\alpha \geq 0)$ satisfying

$$
\begin{aligned}
\left\|x_{i}-\hat{y}_{i}^{(\alpha)}\right\| & <\epsilon_{\alpha}(i=0, \ldots, k-1) \\
\hat{y}_{i}^{(\alpha)} & =\hat{P}_{\alpha} \hat{y}_{i}^{(\alpha)} \hat{P}_{\alpha}+\beta_{i} \hat{P}_{\alpha}^{\perp}(i=0, \ldots, k-1) \\
\operatorname{dim}\left(\hat{P}_{\alpha}(\mathcal{H})\right) & =\hat{N}_{\alpha}=2 k\left[\left(\frac{C(\alpha+1)^{4}}{\epsilon^{2}}\right)^{p}\right] .
\end{aligned}
$$

We now consider the sequence $\mathcal{X}^{(\alpha)}=\Theta^{\alpha}(\mathcal{X})$ and $\mathcal{Y}^{(\alpha)}=\Theta^{\alpha}\left(\hat{\mathcal{Y}}^{(\alpha)}\right)$. Since $\Theta$ is not norm increasing

$$
\left\|x_{i}^{(\alpha)}-y_{i}^{(\alpha)}\right\|<\epsilon_{\alpha}(i=0, \ldots, k-1)
$$

still holds. Furthermore, because

$$
\Theta(|\xi\rangle\langle\chi|)=\left|u_{0} \xi\right\rangle\left\langle u_{0} \chi|+| u_{1} \xi\right\rangle\left\langle u_{1} \chi\right|,
$$

there exists a sequence of projections $P_{\alpha}$ such that

$$
y_{i}^{(\alpha)}=P_{\alpha} y_{i}^{(\alpha)} P_{\alpha}+\beta_{i} P_{\alpha}^{\perp}(i=0, \ldots, k-1)
$$




$$
\operatorname{dim}\left(P_{\alpha}(\mathcal{H})\right)=N_{\alpha}=2^{\alpha+1} k\left[\left(\frac{C(\alpha+1)^{4}}{\epsilon^{2}}\right)^{p}\right] .
$$

From this we can conclude that the density matrix $\rho\left[\mathcal{Y}^{(n-1)} \circ \cdots \circ \mathcal{Y}^{(0)}\right]$ will be living on a subspace of dimension bounded by

$$
1+\sum_{\alpha=0}^{n-1} N_{\alpha} \leq 2+k 2^{n}\left(\frac{C}{\epsilon^{2}}\right)^{p} \sum_{\alpha=0}^{n-1}(\alpha+1)^{4 p} \leq 2+k 2^{n}\left(\frac{C}{\epsilon^{2}}\right)^{p} n^{4 p+1}
$$

and hence

$$
\lim _{n \rightarrow \infty} \frac{1}{n} \mathrm{H}_{\left(\omega_{0}\right)}\left[\mathcal{Y}^{(n-1)} \circ \cdots \circ \mathcal{Y}^{(0)}\right] \leq \lim _{n \rightarrow \infty} \frac{1}{n} \log \left(2+k 2^{n}\left(\frac{C}{\epsilon^{2}}\right)^{p} n^{4 p+1}\right) \leq \log 2 .
$$

Since we can make the right-hand side of the estimate of lemma 1 arbitrary small, this finishes the proof.

LEMma 7. $\mathcal{X} \mapsto \mathrm{h}_{\left(\Theta, \omega_{0}\right)}[\mathcal{X}]$ reaches its upperbound $\log 2$ on the partition $\mathcal{X}=\left(x_{0}, x_{1}\right)$ given by

$$
\begin{aligned}
& x_{k}=\frac{1}{\sqrt{2}}\left(\mathbb{I}+(\mathrm{i}-1)\left|\xi_{k}\right\rangle\left\langle\xi_{k}\right|\right) \\
& \xi_{k}=\frac{1}{\sqrt{2}}\left(\psi^{0}+(-1)^{k} \psi^{1}\right) \quad k=0,1 .
\end{aligned}
$$

From the last two lemmas we can conclude:

THEOREM 4. The dynamical entropy of $\Theta$ with respect to the state $\omega_{0}$ is

$$
\mathrm{h}_{\left(\Theta, \omega_{0}\right)}=\log 2 \text {, }
$$

where the partition supremum is taken over the $\mathcal{L}_{p}$ perturbations of unity.

Acknowledgments. We wish to thank Mark Fannes for very helpful discussions. M. De Cock acknowledges financial support from FWO-project G.0239.96.

\section{References}

[1] R. Alicki, J. Andries, M. Fannes and P. Tuyls, An algebraic approach to the Kolmogorov-Sinai entropy, Rev. Math. Phys. 8(2) (1996), 167-184.

[2] R. Alicki and M. Fannes, Defining quantum dynamical entropy, Lett. Math. Phys. 32 (1994), 75-82.

[3] J. Andries, M. De Cock and M. Fannes, Preprint K.U. Leuven TF-97/29.

[4] G.G. Emch, H. Narnhofer, G.L. Sewell and W. Thirring, Anosov actions on noncommutative algebras, J. Math. Phys. 35(11) (1994), 5582-5599.

[5] B. Simon, Trace ideals and their applications, London Mathematical Society Lecture Notes Series 35, Cambridge University Press, Cambridge, 1979.

[6] P. Tuyls, Towards Quantum Kolmogorov-Sinai Entropy, Ph. D. Thesis, K.U. Leuven, 1997. 